International Journal of Dentistry and Oral Science (IJDOS)

ISSN: 2377-8075

\title{
Role of Stem Cells in Dental Therapy: Recent Trends and an Overview
}

Kumar $\mathrm{S}^{1^{*}}$, Rani $\mathrm{V}^{2}$, Krishna Prasad Biswas ${ }^{3}$

${ }^{1}$ Associate Professor, Department of Dentistry, Indira Gandhi Institutes of Medical Sciences, Sheikhpura, Patna, Bihar, India.

${ }^{2}$ Assistant Professor, Department of Physiology, Patna Medical College and Hospital, Patna, Bihar, India.

${ }^{3}$ Postgraduate Student, Department of Endodontic \& Conversative Dentistry, Government Dental College and Hospital, Chennai, Tamilnadu, India.

\section{Abstract}

Stem cells are the master cells of the body. Stem cell biology is an emerging field of research, provides promising methods in vitro as well as in vivo in animal models which make speculation about a future application in human. The applications in dentistry are under trial and uses in medical field are restricted to certain diseases. The different types and source of stem cells are discussed. Adult dental ecto-mesenchymal stem cells seem promising for future therapy. Human stem cells have been isolated from the dental pulp, exfoliated deciduous teeth, the periodontal ligament, the dental follicle and the dental papilla. The retrieval of dental stem cells is easy and has less ethical issues than medical recovery. Stem cell markers such as STRO-1 were used for the characterization and isolation of stem cells. Adult dental stem cells can differentiate into many dental components, such as dentin, periodontal ligament, cement and dental pulp tissue, but not into enamel.

Aim and Objectives: The objective of this article is to find the recent trends and an overview of the stem cell research concerning fields relevant for dentistry.

Material and Methods: Most of the recent journal articles, lectures notes of renowned speaker and or/stem cell foundation key notes etc., were searched pertaining to know the role of dental stem cells in dental and medical therapy.

Summary: The dental professional will play an important role in both the recovery and the use of these stem cells in both dental and medical regenerative therapies. The regeneration of enamel is still an enigma and poses real test to stem cells science. In future syringe loaded with tissues of stem cells will be available for, like regenerative calcium hydroxide paste and liquid used for regenerative therapy for lost tissues of dental apparatus for healing and repair.

Keywords: Dental Stem Cells; Physiology of Stem Cells; Scope and Source of Stem Cells; Banking of Stem Cells.

\section{*Corresponding Author:}

Dr. Sanjay Kumar,

Associate Professor, Department of Dentistry, Indira Gandhi Institute of Medical Sciences, Sheikhpura, Patna: Pin code: 800014, Bihar, India. Tel: 91-09431383476

E-mail: devasthanam786@yahoo.co.in

Received: May 29, 2015

Accepted: July 20, 2015

Published: August 04, 2015

Citation: Kumar S, Rani V, Krishna Prasad Biswas (2015) Role of Stem Cells in Dental Therapy: Recent Trends and an Overview. Int J Dentistry Oral Sci. 2(8), 126-130. doi: http://dx.doi.org/10.19070/2377-80751500027

Copyright: Kumar $\mathbf{S}^{\circ} 2015$. This is an open-access article distributed under the terms of the Creative Commons Attribution License, which permits unrestricted use, distribution and reproduction in any medium, provided the original author and source are credited.

\section{Introduction}

$[1,2]$ First successful bone marrow transplant was conducted in the year 1956. Embryonic stem cells are isolated from rat blastocysts in the year 1981. The first human embryonic stem cells are isolated in the year 1998 [3-5]. The term Stem cell was proposed by Russian histologist Alexander Maksimov in 1908 at congress of hematologic society in Berlin. In 2005 National institutes of health, USA (NIH) announces discovery of DPSCs by Dr. Irina Kerkis. In 2006 Dr. Kerkis reported discovery of immature dental pulp stem cells (IDPSC), a pluripotent sub-population of DPSC using dental pulp organ culture. In 2003 Dr. Songtao Shi, Pedodontist discovered dental pulp stem cells by utilizing the primary teeth of his daughter and named as he as stem cells from human exfoliated deciduous teeth (SHEDS). In 1988, the National institutes of health and National Science foundation published this definition of "Tissues Engineering". Tissue engineering is positioned to capture the fruit of stem cells, polymer chemistry, materials science, molecular biology and genetics, nano-materials and with the input of clinicians, towards the regeneration of tissues and organs. It offers a unique approach that helps bridge the gap between laboratory discovery and clinical applications [2, 6-8]. The stem cells and tissue engineering have given rise to separate branch termed as "regenerative dentistry". In about the $5^{\text {th }}$ embryonic week, odontogenesis is induced from the oral epithelium; the underlying mesenchyme of the tooth papilla is responsible for the regulation and differentiation of these cells as well as the control of crown and root formation. Over 200 regulatory genes are involved in odontogenesis cells communicate via signals molecules and growth factors predominantly growth from the four eminent families, fibroblast growth factors- $\beta$ (FGF- $\beta$ ), Hedgehog wingless and transforming growth factors- $\beta$ (TGF- $\beta$ ) to which the bone morphogenic protein (BMPs) also belong, are important in the regulation of odontogenesis. 
Wisdom teeth develop postnatally; their enamel organ has formed by about the $72^{\text {nd }}$ month of life. This means that up to that point, undifferentiated dental embryonic tissue exists in the jaws. The development of the third molars is the only organogenesis which takes place completely after birth. As opposed to human teeth, the incisors of rodents grow throughout the animal's life source of epithelial stem cells, apical bud cells (ABCs), in the apical epithelium is responsible for continuous enamel production. The homeostatic microenvironment in this region provides stem cells with a space in which they can develop. Dentistry has long exploited the life-long regeneration potential of adult stem cells in human dental pulp which give rise to tertiary dentin, therapeutically employed for direct and indirect pulp capping after caries/ accidental pulp exposure of teeth. The application of calcium hydroxide or calcium phosphate, among other substances, can induce pulpal progenitor cells to differentiate into dentinoblasts. Stem cells could change dentistry in two ways. One is that entire teeth can be grown out of stem cells in vitro. The other method involves reattaching your existing teeth to your jawbone with the use of stem cells in case periodontally loose teeth due to periodontal diseases and or trauma. The tooth will anchor and fuse to its previous environment and the stem cells will turn into cementum, gingival lining and/or whatever necessary cells are needed.

\section{Classification}

There are two types of stem cells 1. Embryonic Stem Cell, 2. Adult Stem Cell.

Single embryonic stem cell has the potential to differentiate into all 220 types of specialized cells that make up the human body. This ability to grow and regenerate tissues is the focus of the emerging field of personalized medicine which uses a patient's own stem cells for biologically compatible therapies. Adult stem cells are responsible for the regeneration and replacement of tissue damaged by disease or injury. There are two properties of stem cells that make it different from any other specialized cells in the body. These properties are self- renewal - the ability to go through numerous cycles of cell division while maintaining their undifferentiated state and the ability to differentiate into a specialized cell type. Another unique property of stem cells is their ability to grow in vitro - outside of the body. In the right culture medium and under the right controlled conditions, stem cells are able to proliferate indefinitely. The expansion of stem cells in this controlled tissue culture and by maintaining them in their undifferentiated state leads to the development of specific stem cell lines. These stem cell lines are invaluable for present day stem cell research and medical therapies $[2,8,9]$. Characteristics of stem cells are 1.Totipotency: generate all types of cells including germ cells. 2. Pluripotency: generate all types of cells except cells of the embryonic membrane 3. Multipotency: differentiate into more than one mature cell (msc) 4. Self-renewal: divide without differentiation and create everlasting supply. Plasticity: mscs have plasticity and can undergo differentiation. The trigger for plasticity is stress or tissue injury which upregulates the stem cells and releases chemoattractants and growth factors. Embryonic stem cells that are harvested from the first $50-150$ cells of a $4-5$ days old fertilized egg or blastocyst. Embryonic stem cells are the result of the fertilization of an egg by a sperm. Cells produced by the first few divisions of the fertilized egg are totipotent. These cells can differentiate into embryonic and extra-embryonic cell types. Embryonic stem cells are the cells that make up the human body and extra-embryonic will make up the placenta. Pluripotent stem cells are those embryonic stem cells that are harvested after the $5^{\text {th }}$ day from the inner mass of this blastocyst (ref. Figure.no.1). These pluripotent stem cells go on to differentiate into any of the three

Figure 1. Physiological embryogenesis and odontogenesis [Source-Schweiz Monatsschr Zahnmed Vol.120 10/2010 Figure modified and redrawn].




types of cells that derive the germ layers: ectoderm, endoderm and mesoderm. The adult stem cells are present in a foetus and also in humans after birth. The adult stem cells are found deep within organs and tissues and are spread diffusely throughout. Adult stem cells to replace lost cells, stem cells typically generate intermediate cells called precursor or progenitor cells, which are no longer capable of self-renewal. However, they continue undergoing cell divisions, coupled with maturation, to differentiate into fully specialized cells. Adult stem cells are usually designated according to their source and their potential. Adult stem cells are multipotent because their potential is normally limited to one or more lineages of specialized cells. However, a special multipotent stem cell that can be found in bone marrow and dental pulp called the mesenchymal stem cell can produce all cell types of bone, cartilage, fat, muscle, and connective tissues. However, a number of recent studies show that stem cells from one area may be manipulated to grow into cells types of a completely different tissue. This ability is called transdifferentiation or plasticity and different types of adult stem cells have varying degrees of plasticity. Modifying the growth medium when stem cells are cultured in-vitro or transplanting them to an organ of the body different from the one they were originally isolated from can induce plasticity $[1,2,10,11]$.

\section{Stem cell markers}

Stem cell markers help to identify characterize, and isolate stem cells. STRO-1, a trypsin-resistant cell-surface antigen, is a commonly used dental stem cell marker for all dental MSCs. Researcher uses stem cell markers to distinguish these rare cells. The stem cell markers are coating of the surface of every cell in the body are specialized proteins, called receptors that have the capability of selectively binding or adhering to other "signaling" molecules. There are many different types of receptors that differ in their structure and affinity for the signaling molecules. These same cell surface receptors are the stem cell markers. Each cell type, for example a muscle cell, has a certain combination receptors on their surface that makes them distinguishable from other kinds of cells. The uniqueness of these receptor allow scientist to mark these stem cells. Receptors of stem cell markers are given shorthand names based on the molecules that bind to the stem cell surface receptors. For the mesenchymal stem cells it is the Stro $1+$ antigen. Dental stem cells are the most accessible stem cells; they are isolated from the dental pulp of healthy teeth, periodontal ligament including the apical region of developing teeth and other tooth structures. Craniofacial stem cells, including dental stem cells, originate from neural crest cells and mesenchymal cells during development. Neural crest cells share the same origin as progenitor cells that form the neural tissue. Adolescents have two excellent opportunities for banking their stem cells from extracted teeth: following extraction of bicuspid teeth for orthodontic treatment and when their wisdom teeth are extracted. The bicuspid teeth are not fully formed until between the ages of 12 to 14 years. The apex of the root is not fully closed, which ensures a good blood supply and more proliferation of stem cells within the pulp. The same scenario is true with wisdom teeth. The roots of the wisdom teeth are not fully formed until after the age of 18 years, extracting these teeth during the teenage years helps to ensure the greatest abundance of proliferative stem cells. The follicular tissue of an unerupted tooth may also prove to be a valuable source for stem cells. The follicle is found at the coronal portion of impacted teeth. This soft tissue lining is ecto-mesenchymal origin and is responsible for producing the enamel portion of the tooth. This soft tissue is removed and discarded along with the impacted tooth. The stem cells that are found in the pulp of deciduous and permanent teeth are adult multipotent mesenchymal stem cells. The central region of the pulp contains large nerve trunks and blood vessels. This area is lined peripherally by a specialized odontogenic area which has three layers; 1. Cell rich zone: innermost pulp layer which contains fibroblasts and undifferentiated mesenchymal stem cells. These cells are dispersed diffusely throughout this layer and the number of stem cells depends upon the quantity of pulpal tissue (Age, the type of tooth, incisor vs. molar, deciduous vs. permanent, stage of development or resorption) 2. Cell free zone (zone of Weil): This is rich in both capillaries and nerve networks. The nerve plexus of Rashkow is located 3. Odontoblastic layer: Outermost layer which contains odontoblasts and lies next to the predentin and mature dentin. Other cells found in the dental pulp include fibroblasts (the principal cell), odontoblasts, defense cells like histiocytes, macrophages, granulocytes, mast cells and plasma cells. Pulpectomies on vital pulps is another accessible means to collect viable stem cells. Dr. Songtao Shi, Pedodontist, have discovered a unique type of mesenchymal stem cell in the dental pulp of deciduous teeth. Stem cells from deciduous teeth, which are formed during the embryonic phase of human development, nicknamed 'SHED" (stem cells from human exfoliated deciduous teeth); he believe that these stem cells behave differently than adult stem cells. SHED cells are capable of extensive proliferation and differentiation, which makes them an important resource of stem cells for the regeneration and repair of craniofacial defects, tooth loss and bone regeneration. Indeed, dental stem cells from the deciduous tooth have been induced to express neural markers such as nestin. Similarly, bone marrow derived stem cells also have been induced to express neural cell markers. The expression of neural markers in dental stem cells elicits the imagination of their potential use in neural regeneration such as in the treatment of Parkinson's disease. In balance, the potential of dental stem cells in both dental and non-dental regeneration should be further explored. The periodontal ligament serves as a reservoir for many cell types, including fibroblasts, Osteoblasts/clasts, cementoblasts/clasts, and odontoclasts. The remarkable characteristic of the periodontal ligament is its ability to regenerate and repair virtually every other tissue type that comprises the periodontium. Undifferentiated mesenchymal cells of the periodontal ligament can differentiate into osteoblasts, chondrocytes and adipocytes. New research has shown that post-natal stem cells are also found in the periodontal ligament. Ligament removed from extracted third molars transplanted into culture yielded multiple rapidly dividing colonies of stem cells that expressed specific proteins characteristic of postnatal stem cells. The replication rate of these stem cells was similar to that of dental pulp stem cells. Following transplantation into mice, these stem cells produced cementum, periodontal ligament and fibrous structures similar to sharpey's fibers, which anchor cementum to bone. The other sources of stem cells accessible from the oral cavity during oral surgical procedures are form alveolar bone: The periosteum covers most of the bones in the body. The periosteum is a very dense, tough layer of fibrous tissue intended to act as a covering for bone and provide progenitor cells for bone growth and repair. Periosteum is divided into an outer "fibrous layer" and inner "cambium layer". The fibrous layer contains fibroblasts while the cambium layer contains progenitor cells which develop into osteoblasts. Cultured adult human periosteal stem cells demonstrate mesenchymal multipotency, suggesting that they may be used to repair tissue, upon enzymatic release and culture expansion, cells harvested from the periosteum can give rise to cartilage and bone. The oral cavity contains 
masticatory mucosa, such as gingiva, and lining mucosa, such as buccal mucosa. Oral mucosal epithelium has drawn attention as a cell source for a variety of tissue engineered reconstructions, such as oral cavity, epidermis, and especially ocular surface reconstruction and the transplantation of human oral epithelial sheets grown on various substrates can be useful for tissue reconstruction. In the clinical setting, the quality of the cultivated graft is the key to success. Bone tissue engineering is a promising approach for bone reconstruction in oral-maxillofacial surgery. The bone graft that is being harvested and transplanted contains stem cells in addition cortical and particulate bone. In the future small quantities of patients' bone will be harvested, transported to a laboratory and expanded to produce larger quantities of bone. This particulate bone graft will be implanted into the donor. Because this is an autologous graft the potential for rejection will be minimal. The non-dental sources of stem cells are bone marrow derived mesenchymal stem cells. A bone marrow transplant involved a donor and recipient with a close cellular match. Mesenchymal stem: cells are often viewed as a yardstick of adult stem cells. Presently peripheral blood stem cell collection is being used in place of bone marrow aspiration. Adipose-derived stem cells have also been isolated from human fat, usually by method of liposuction. This cell population seems to be similar in many ways to mesenchymal stem cells (MSCs) derived from bone marrow. However, it is possible to isolate many more cells from adipose tissue and the harvest procedure itself is less painful than the harvest of bone marrow. Umbilical cord stem cells: derive from the blood of the umbilical cord. There is a growing interest in their capacity for self replication and multi-lineage differentiation. Umbilical cord stem cells have been differentiated into several cell types such as cells of the liver, skeletal muscle, neural tissue and immune cells. Their high capacity for multi-lineage differentiation is likely attributed to the possibility that umbilical cord stem cells are chronologically closer derivatives of embryonic stem cells than adult stem cells. Several studies have shown the potential of umbilical cord stem cells in treating cardiac and diabetic diseases. Amniotic fluid-derived stem cells can be isolated from aspirates of amniocentesis during genetic screening or collection at the time of delivery $[2,9,10,12,13]$.

\section{Banking stem cells from teeth}

Banking of tooth for retrieval of stem cells are more recent trends and are more prevalent in developed countries like Japan, China and in western countries. Tooth-derived stem cells are readily accessible, and provide an easy and minimally invasive way to obtain and store stem cells for future use. Banking teeth and tooth-derived stem cells is a reasonable and simple alternative to harvesting stem cells from other tissues requiring invasive surgical procedures, and does not pose the ethical problems associated with embryonic stem cell harvesting. Stem cells can be obtained from loose baby teeth and from extracted permanent teeth. Tooth extractions are common prior to beginning orthodontic treatment or at the time the wisdom teeth are removed. Store this convenient source of stem cells. If needed, using your own stem cells (autologous donation) poses fewer risks for rejection following transplantation. Teeth that are diseased or infected are not good sources for stem cell retrieval and storage. The tooth is transferred into a sample vial containing a buffered saline solution. A recently extracted tooth is at a temperature of 98.6 degrees Fahrenheit. Hypothermia is induced by placing the tooth in the solution at room temperature (74 degrees Fahrenheit). Hypothermia is the temperature at which cellular metabolism is slowed down and this is a temperature below 92 degrees Fahrenheit. The specimen vial is placed into an insulated transport vessel. To maintain hypothermia during transport and to prevent spikes in temperature the sample vial is placed into a thermette. The thermette consists of a phase change material that absorbs heat to maintain the internal ambient temperature of the transport vessel below 90 degrees Fahrenheit. No preparation or refrigeration of the kit is necessary. The kit should be kept at room temperature and in a dry place. The transport vessel is placed into an insulated shipping box. The insulated box adds another layer of temperature protection during the transport of the tissue sample. A security seal is placed on the front cover of the assembled transport kit. By maintaining the tissue in a hypothermic state the kit needs to be delivered to the processing facility for cryopreservation within $48 \mathrm{hrs}$ of the tooth being extracted. Care is taken not to apply the sterilizing agent directly to the exposed tissue. The tooth is cracked open and the pulpal tissue is removed and assessed for viability. The tissue is then placed through several washes using an antibiotic solution. After each wash the sample is centrifuged to remove any cellular debris. Once the tooth arrives at the processing facility the tooth is rinsed several times with an antibiotic solution. The tooth is sterilized with by wiping the external surface of the tooth avoiding any exposed pulpal tissue. The harvesting of the dental stem cells from a tooth is conducted in a sterile environment, following an established protocol. The pulp is removed from the inside of the tooth using a variety of manual techniques. In most case the tooth is "cracked" open and the pulp is removed from the tooth portions using dental curettes. The tissue is assessed for viability. Only viable tissue goes on to cryopreservation. The pulpal tissue is washed several times in an antibiotic solution. The pulpal tissue is cryopreserved in its native state. The tissue and stem cells are minimally manipulated and are not enzymatically treated or expanded in cell culture. The cells go through a final wash and centrifuge to remove any cellular debris prior to cryopreservation. Cryopreservation is a process where cells or whole tissues are preserved by cooling to low subzero- temperatures, such as (typically) $-196^{\circ} \mathrm{C}$ (the boiling point of liquid nitrogen). At these low temperatures, any biological activity, including the biochemical reactions that would lead to cell death, is effectively stopped. However, when vitrification solutions are not used, the cells being preserved are often damaged due to freezing during the approach to low temperatures or warming to room temperature. The risks of cryopreservation are the phenomena which can cause damage to cells during cryopreservation. In clinical cryopreservation, vitrification usually requires the addition of cryoprotectants prior to cooling. The cryoprotectants act like antifreeze: they lower the freezing temperature. They also increase the viscosity. Instead of crystallizing the syrupy solution turns into an amorphous ice i.e. it vitrifies. In cryopreservation, the solute such as dimethyl sulfoxide a common cryoprotectant must penetrate the cell membrane in order to achieve increased viscosity and depressed freezing temperature inside the cell. The drawback is that the cryoprotectants are often toxic in high concentration.

The liquid nitrogen storage tanks are connected to automatic liquid nitrogen replenishes lines. Each storage tank is monitored separately. The storage facility is required to have emergency backups in place in case of a power outage. The sample vials are placed in boxes. The boxes are numbered and placed into vertical racks. The numbered racks are submerged in liquid nitrogen. Each box and rack are included in a database, the sample vial can be referenced against this database to identify its location in a particular liquid nitrogen tank. The liquid nitrogen levels are con- 
tinuously monitored within the tanks. The tank is prefilled with liquid nitrogen prior to placement of the racks. Placement of the racks into the tanks causes displacement of the liquid nitrogen. Pre-measuring prior to placement of the racks prevents under filling or overflowing of the liquid nitrogen. Each tank is monitored for temperature fluctuations as well as changes in the liquid nitrogen levels. Whenever a rack needs to be removed from a liquid nitrogen storage tank, strict protocols are followed that prevents temperature changes to the cryopreserved tissues. The rack is removed from the storage tank and transferred to a vat filled with liquid nitrogen. This vat can be moved to a bench top from where the samples are added to or removed from the rack. Performing this stepped transfer also prevents the cover of the storage tank from being open for any extended period of time and avoids the loss of liquid nitrogen by evaporation. The optimal way to grow cells is to employ a bioreactor. A bioreactor may refer to any device or system that supports a biologically active environment. Bioreactors allow for precise and continuous control of culture conditions and also allow for introduction of different stimuli to tissue cultures.

Steps in the dental clinic for preparation of a tooth for stem cells (in brief): Examine the tooth and rule out any infection, rinse the tooth with antibiotics/saline solution, transfer to transportation tube/kit, add saline solution, wait for five minutes, Seal the tube and transport under room temperature (hypothermia) before 48 hrs. The steps in the laboratory as follows: prepared tooth is cracked open and the pulp is removed with the dental curette, identification of stem cells with markers, separation of viable cells by centrifuge, cryopreservation and retrieval. Magnetic freezing is the newer method, Cell alive system (CAS). Under the condition of CAS magnetic field energy, water clusters do not accumulate but remain in smaller groups, thus minimizing restraining the expansion of the water. This technology is called CAS and uses the phenomena that applying even a weak magnetic field to water or cell tissue will lower the freezing point of that body by up to 6-7 degrees Celsius. Using CAS, Hiroshima University claims that it can increase the cell survival rate in teeth to as high as $83 \%$. Maintaining a CAS system is a lot cheaper than cryogenics and more reliable as well [13-16].

The stem cells uses in the dentistry are as follows, regeneration of damaged coronal dentin and pulp, regeneration of resorbed root, cervical or apical dentin, and repair perforations, periodontal regeneration, repair and replacement of bone in craniofacial defects, whole tooth regeneration. A promising approach to tooth regeneration in animal experiments may be to obtain epithelial stem cells from third molars of newborn or juvenile animals according to many studies. The potential uses for stem cells in medical field as follows, presently all of the current therapies involve adult stem cells and these are cell-based therapies. Transplanting or "grafting" tissue to combat shortages of donated organs seems to be high on the list of priorities. The treating diseases such as Alzheimer's, Parkinson's, spinal cord injury, stroke, burns heart disease, arthritis and diabetes. Drugs are being applied directly to human cells and this will provide more relevant data than drug testing on animals. Testing medications for safety on differentiated cells from pluripotent cell lines, as anti-cancer drugs are now tested on cancer stem cell lines. Understanding cell division and development: Stem cells are also providing a means to better understand particular diseases of genetic origin through studying cells with those mutations.

\section{Summary}

Current research on dental stem cells therapy is expanding. Stem cell biology in dentistry will emerge to become an important aspect in the everyday practice of dental professionals in near future. Patients come to the dentist because of dental/oral infections, trauma, congenital anomalies or other diseases such as oro-facial cancer and salivary gland disorders. Dental caries and periodontal disease remain two of the highly prevalent dental diseases involving the dental tissues. Dental restorative materials like amalgam, composites, and even titanium dental implants have limited shelf life. It has not yet been possible to find a source of human adult ecto-dermal stem cells to regenerate enamel post-eruptively. The autologous tooth regeneration and implantation in patient is still an enigma and matter of research. In future syringe loaded with tissues of stem cells will be available for, like regenerative calcium hydroxide paste and liquid used for regenerative therapy for lost tissues of dental apparatus for healing and repair.

\section{References}

[1]. Ulmer FL, Winkel A, Kohorst P, Stiesch M (2010) Stem cells: prospects in dentistry. Schweiz Monatsschr Zahnmed 120(10): 860-869.

[2]. Gregory C (2015) Stem cells: Emerging medical and dental therapies and the dental professional. President - StemSave, Inc. 1-17.

[3]. Singh H, Bhaskar DJ, Rehman R, Jain CD, Khan M (2014) stem cells: An Emerging future in dentistry. Int J Adv Health Sci 1(2): 17-23.

[4]. Marwar PP, Mani A, Sachdev S, Sodhi NK, Anju A (2012) Stem cells in dentistry: An overview. Parvara Med rev 4(2): 11-15.

[5]. Sharma R, Bhargava D, Yadav M, Rastogi P, Chandavarkar V, et al. (2013) Dental stem cells harnessing newer possibilities. J Indian Acad Forensic Med 35(4): 378-382.

[6]. Tatullo M, Marrelli M, Shakesheff KM, White LJ (2014) Dental pulp stem cells: function, isolation and applications in regenerative medicine. J Tissue Eng Regen Med DOI: 10.1002/term.1899.

[7]. Lesot H, Brook AH (2009) Epithelial histogenesis during tooth development. Arch Oral Biol 54: S25-33.

[8]. Sakai VT, Zhang Z, Dong Z, Neiva KG, Machado MAAM (2010) SHED differentiates into functional odontoblasts and endothelium. J Dent Res 89(8): 791-796.

[9]. Arora V, Arora P, Munshi AK (2009) Banking stem cells from human exfoliated deciduous teeth (SHED), saving for the future. J Clin Padiat Dent 33(4): 289-294.

[10]. Masthan KMK, Aravindha B, Leena S, Gopala Krishnan T (2012) Teeth as a life bank (Stem cells in dentistry), review article. J Medicine and Medical Sciences 3(7): 456-458.

[11]. Roopa RN (2009) Stem cell therapy - Hype or hope? A review. J Conserv Dent 12(4): 131-138.

[12]. Dziubiniska P, Jasolska M, Przyborowska P, Adamiak Z (2013) Stem cells in dentistry- review of literature. Polish journal of veterinary sciences 16(1): 135-140.

[13]. Hegde MN, Hegde P, D'Souza CM (2012) Tooth Stem Cell Banking-A Review. Conservative and Endodontic Society of Kerala 45.

[14]. Wang SL, Wang XJ (2008) Tooth regeneration-dreams to reality. West China journal of stomatology 26(2): 115-117.

[15]. Morsczeck C, Reichert TE, Völlner F, Gerlach T, Driemel O (2007) The state of the art in human dental stem cells research. Mund Kiefer Gesichtschir 11(5): 259-266.

[16]. Ikeda E, Morita R, Nakao K, Ishida K, Nakamura T, et al. (2009) Fully functional bioengineering tooth replacement as an organ replacement therapy. Proc Natl Acad Sci U S A 106(32): 13475-13480. 